Bundesgesundheitsbl 2020 63:891-897 https://doi.org/10.1007/s00103-020-03165-0 Online publiziert: 15 . Juni 2020

(c) Der/die Autor(en) 2020

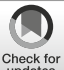

\author{
Astrid Blaschek ${ }^{1,3} \cdot$ Katharina Vill $^{1}$ ' Wolfgang Müller-Felber ${ }^{1} \cdot$ Ulrike Schara $^{2}$ \\ 'Abteilung Pädiatrische Neurologie, Entwicklungsneurologie und Sozialpädiatrie, Dr. von Haunersches \\ Kinderspital, Ludwig-Maximilians-Universität München, München, Deutschland \\ ${ }^{2}$ Abteilung Pädiatrische Neurologie, Universitätskinderklinik, Universitätsklinikum Essen, Essen, \\ Deutschland \\ ${ }^{3}$ München, Deutschland
}

\section{Molekulare Therapien bei neuromuskulären Erkrankungen im Kindesalter - Große Hoffnungen und unbekannte Risiken}

\section{Einleitung}

Neuromuskuläre Erkrankungen stellen eine heterogene Gruppe monogenetischer Erkrankungen dar, die häufig schon in der Kindheit Symptome zeigen. Die spinale Muskelatrophie (SMA) und Muskeldystrophie Duchenne (DMD) zählen zu den am häufigsten auftretenden seltenen Muskelerkrankungen im Kindesalter. Die Therapieoptionen für einige seltene monogenetische neuromuskuläre Erkrankungen haben sich im letzten Jahrzehnt entscheidend verändert. Großen Anteil daran haben neue molekulare Therapieansätze, die den direkten gentherapeutischen Ersatz des fehlenden Gens ermöglichen oder eine alternative Prozessierung des betroffenen Gens bzw. eines verwandten (Pseudo)Gens bewirken. Diese neuen molekularen Therapien bilden den Schwerpunkt dieser Arbeit. Sowohl potenzieller Nutzen als auch mögliche Risiken sollen dargestellt werden.

Grundsätzlich werden drei molekulargenetische Therapieprinzipien verfolgt:

1. Antisense-Oligonukleotid-Technologie u. Ä. (alternatives Prozessieren des betroffenen bzw. verwandten Gens),

2. Read-Through-Therapie (Überlesen eines Stop-Codons),

3. Genersatztherapie.

\section{Antisense-Oligonukleotid- Technologie und „kleine Moleküle"}

Bei der Antisense-Oligonukleotid-Technologie wird die Biosynthese des Zielproteins auf genomischer Ebene über Hochregulation der effektiven Proteinsynthese verändert. Dies geschieht durch Bindung von Antisense-Oligonukleotiden (ASO) oder sog. kleinen Molekülen (Small Molecules) an eine Spleißstelle (Grenze zwischen codierenden und nichtcodierenden Abschnitten der DNA innerhalb eines Gens).

Damit kann sowohl die Transkription eines codierenden Abschnitts (Exons) hochreguliert werden, wie etwa des Exons 7 im Survival-Motor-Neuron-2(SMN2)-Gen in der Therapie einer spinalen Muskelatrophie mit den Wirkstoffen Nusinersen und Risdiplam (- Abb. 1a), als auch die Ablesung eines betroffenen Exons blockiert werden, wie z. B. durch das Überspringen eines Exons (Exon Skipping) in der Therapie mit dem Wirkstoff Eteplirsen bei Muskeldystrophie Duchenne (• Abb. 1b).

Schwierigkeiten bei der Anwendung der Therapie bestehen vor allem aufgrund der notwendigen systemischen Applikation der Medikation bei den Muskeldystrophien. Die eher geringe Bioverfügbarkeit bei systemischer Gabe im Zielgewebe führt $\mathrm{zu}$ dosisbegrenzenden, vor allem renalen und hämatopoetischen Nebenwirkungen und der Notwendigkeit von häufigen und sehr regelmäßigen Gaben.

Da ASO die Bluthirnschranke nicht hinreichend überwinden können, muss bei der spinalen Muskelatrophie die Behandlung durch Injektionen in den Liquorraum (intrathekal) erfolgen. Die Behandlung mit Nusinersen wurde bereits seit 2017 für alle Formen der 5q-assoziierten spinalen Muskelatrophie (SMA) zugelassen. „Kleine Moleküle“ zur Behandlung der spinalen Muskelatrophie werden aktuell in klinischen Studien geprüft [1].

Durch sogenannte Nonsense-Mutationen entstehen im Genom Stop-Codons, welche zum vorzeitigen Abbruch der ribosomalen Proteinbiosynthese während der Translation führen. Dadurch wird ein verkürztes und nichtfunktionales Protein gebildet. Durch das Überlesen eines Stop-Codons auf RNA-Ebene soll wieder ein funktionales vollständiges Protein erzeugt werden ([1]; • Abb. 1c).

Bislang wurde dieses Therapieprinzip vor allem bei Muskeldystrophie Duchenne untersucht und führte 2014 zur europäischen Zulassung der Substanz Atalu-

\section{Read-Through-Therapie}




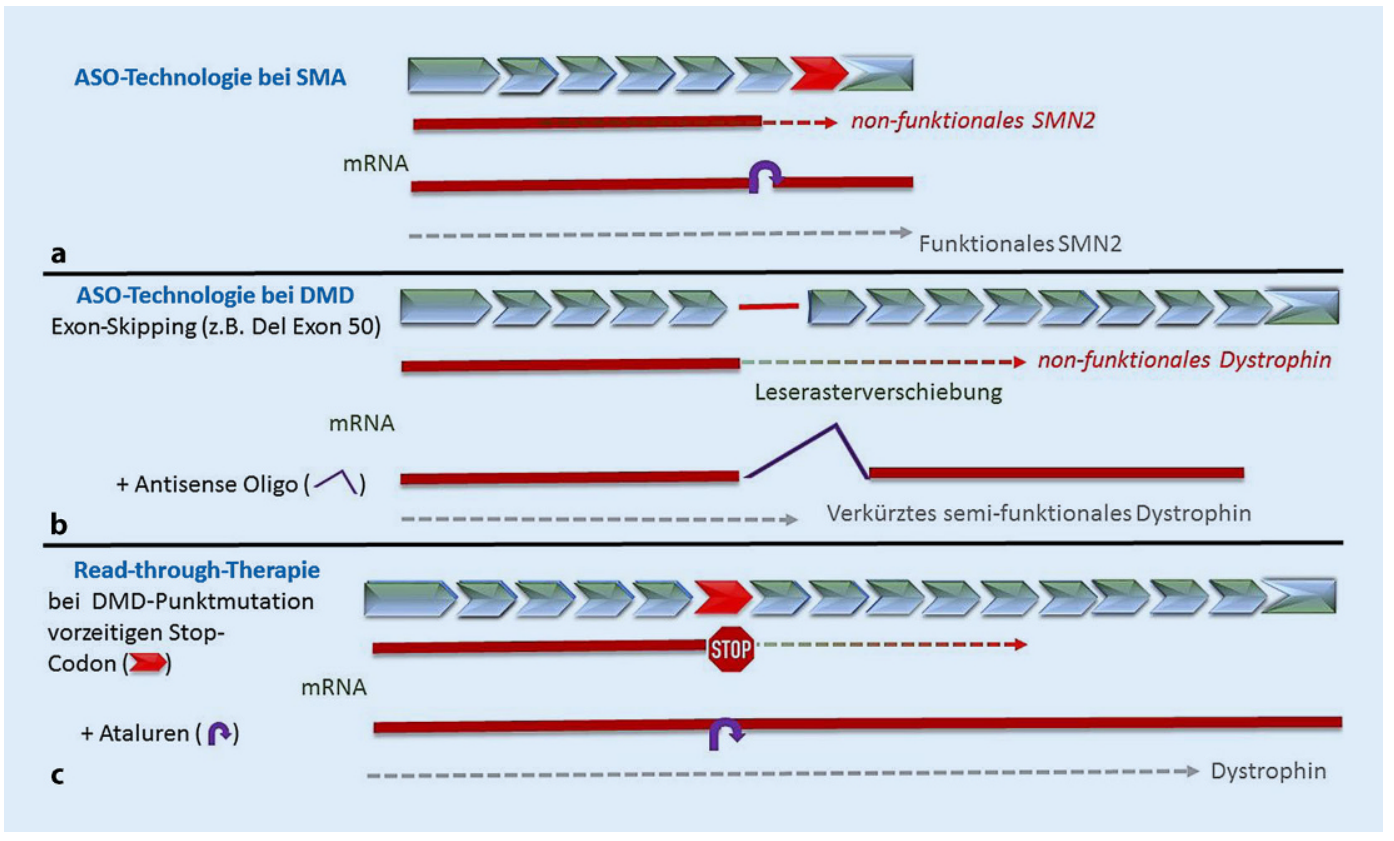

Abb. 1 A Prinzipien der Antisense-Oligonukleotid-Technologie (ASO-Technologie) und der Read-Through-Therapie. a Pathologie: Eine Mutation im SMN2-Gen führt zu alternativem Spleißen und der Herstellung eines non-funktionalen Proteins. Therapie: Das ASO verdeckt die mutierte Stelle und die Transkription des codierenden Abschnitts (Exon 7) wird hochreguliert. b Pathologie: Deletionen von Exonen im Dystrophingen führen zur Leserasterverschiebung und damit zu verkleinerten, funktionslosen Dystrophinfragmenten. Therapie: Beim Exon-Skipping stellen ASO unter Auslassen definierter Exone das Leseraster wieder her. Ein verkürztes, semifunktionales Dystrophin wird erreicht. c Pathologie: Bei vorzeitigem Stop-Codon wird nur ein verkürztes Fragment transkribiert und führt zu einem non-funktionalen Protein. Therapie: Die Substanz Ataluren führt zum Überlesen des Stop-Codons und damit zur vollständigen Transkription von Dystrophin in ganzer Länge

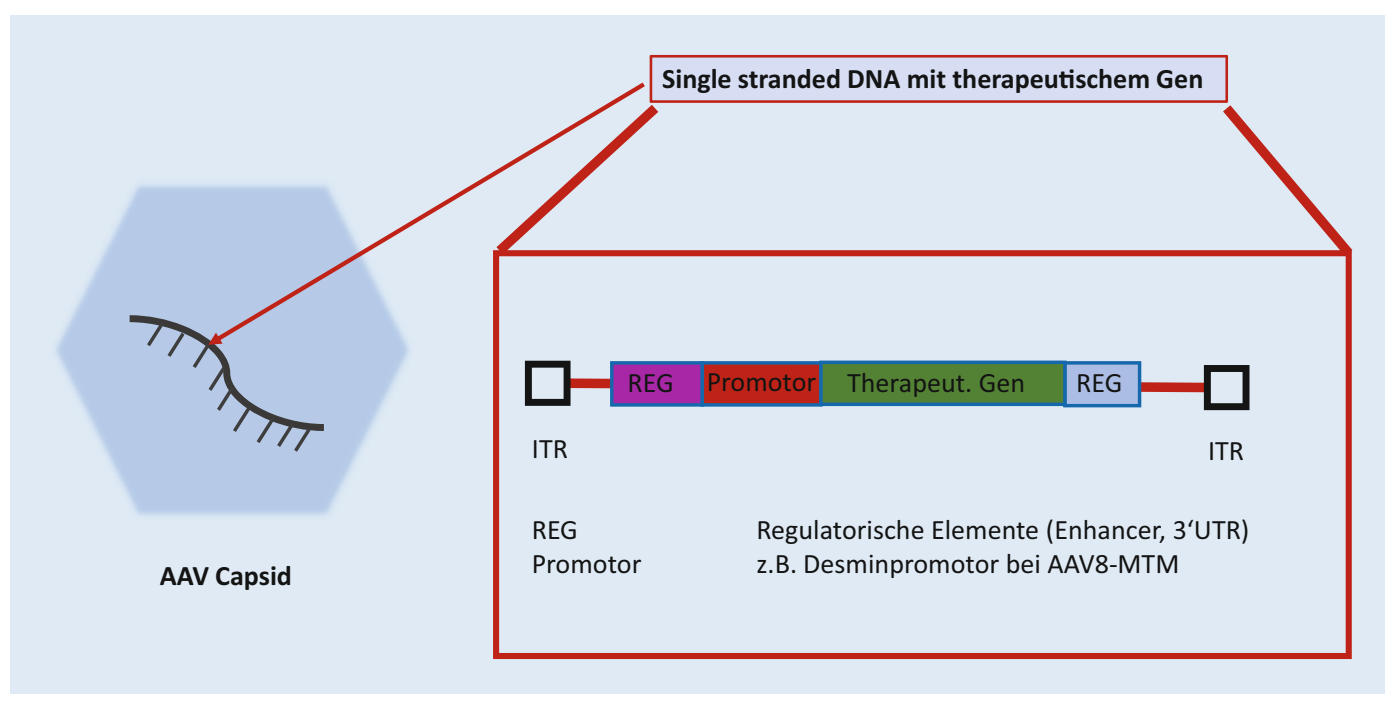

Abb. $2<$ Aufbau eines Trägervirus zur Gentherapie (adenoassoziierter Virus, AAV). Das Vektorkonstrukt liegt im Viruskapsid als selbstkomplementärer Einzelstrang vor. Im Kern der Wirtszelle werden unabhängig von der Transkriptionskapazität der Zelle daraus Doppelstränge synthetisiert. Das Genkonstrukt umfasst neben flankierenden invertierten terminalen Repetitionen (ITR) als Teil der regulatorischen Einheiten (REG), einen zielgewebsspezifischen Promotor sowie das therapeutische Gen

ren. Aktuell laufen weitere Studien u.a. bei Becker-Muskeldystrophie sowie der Dysferlinopathie.

\section{Genersatztherapie}

Virale Vektoren tragen das gewünschte Gen im besten Fall in seiner gesamten Länge (• Abb. 2). In präklinischen Stu- dien sind die Entwickler vor allem mit folgenden Fragen und Hindernissen konfrontiert [2].

a) Welches ist die beste Applikationsform: lokal vs. systemisch vs. spezifische Kompartimente (z.B. Spinalkanal)? Kann bei ZNS-Erkrankungen die Bluthirnschranke überwunden werden? b) Welcher Serotyp des Virusvektors sollte benutzt werden? Dabei spielt vor allem das gewählte Kapsidprotein sowohl für den Tropismus (Affinität zu einer Körperstruktur) als auch für die mögliche Immunreaktion eine entscheidende Rolle.

c) Wie kann die Expression des Zielgens kontrolliert werden? Das genthe- 
rapeutische Konstrukt muss an die limitierte Packkapazität des Trägervirus angepasst werden.

d) Welche Modifikationen können am Zielgen vorgenommen werden (welche Sequenzen sind potenziell verzichtbar?), um der limitierten Packkapazität des Trägervirus Rechnung zu tragen?

e) Wie kann die potenzielle Immunogenität des Vektors bei der humanen Anwendung vorab abgeschätzt bzw. beeinflusst werden? Hierbei spielen sowohl das angeborene unspezifische als auch das adaptive Immunsystem eine Rolle. Neue Vektoren wie der Gutless-Adenovirus (GLAd, HDAd) könnten zukünftig eine Option darstellen, um die Immunogenität der Therapie deutlich zu verringern [3]. Die immunologische Reaktion (v.a. Antikörperproduktion) ist einer der Hauptfaktoren, der bei einer potenziell notwendigen Retherapie problematisch sein wird.

Diese Faktoren bestimmen neben anderen Einflüssen die Effektivität und das Nebenwirkungsprofil des Gentransfers.

Nach der präklinischen Phase folgen erste Anwendungen an betroffenen $\mathrm{Pa}$ tienten. Hier muss vorab aus den tierexperimentellen Daten eine minimal wirksame Virusdosis kalkuliert werden [4]. Daher werden die Phase-1-Studien in der Regel als Dosiseskalationsstudien geführt, um an einem kleinen Kollektiv erste Daten über das humane Nebenwirkungsspektrum bzw. die Immunogenität sowie die wahrscheinlich bestwirksame Dosis zu ermitteln.

Im Folgenden werden die aktuellen, sich in klinischer Studienphase befindlichen Therapien genauer beschrieben, wobei der Schwerpunkt auf die SMA und Muskeldystrophie Duchenne gelegt wird. Gute Übersichten über sich in Erprobung befindliche neue Therapien bei neuromuskulären Erkrankungen finden sich in aktuellen Übersichtsarbeiten [5-8].

\section{Spinale Muskelatrophie}

Die autosomal rezessiv vererbte spinale Muskelatrophie (SMA) ist eine der häufigsten neurodegenerativen Erkrankun-

Bundesgesundheitsbl 2020 -63:891-897 https://doi.org/10.1007/s00103-020-03165-0

(c) Der/die Autor(en) 2020

\section{A. Blaschek $\cdot$ K. Vill $\cdot$ W. Müller-Felber $\cdot$ U. Schara \\ Molekulare Therapien bei neuromuskulären Erkrankungen im Kindesalter - Große Hoffnungen und unbekannte Risiken}

\section{Zusammenfassung}

Seltene neuromuskuläre Erkrankungen, wie spinale Muskelatrophie und Muskeldystrophie Duchenne, sind Erbkrankheiten, die sich bereits im Kindesalter zeigen. Die Therapieoptionen haben sich in den letzten Jahren entscheidend verändert. Großen Anteil daran haben neue molekulare Therapieansätze, die den direkten gentherapeutischen Ersatz des fehlenden Gens ermöglichen oder eine alternative Prozessierung des betroffenen Gens bzw. eines verwandten (Pseudo)Gens bewirken.

Vor allem der Verlauf der $5 q$-assoziierten spinalen Muskelatrophie hat sich durch die Verfügbarkeit solcher kausalen Therapien deutlich verändert, während für die meisten
Muskelerkrankungen die Ergebnisse laufender Studien noch ausstehen. Als erreichbares Therapieziel muss im Bereich der neuromuskulären Erkrankungen die Verlangsamung der Progredienz, aber nicht die vollständige Heilung angesehen werden. Aktuell sind nur limitierte Erfahrungen verfügbar. Insbesondere die langfristige Effektivität und die möglichen Risiken sind noch unbekannt. Daher sollten diese Therapien unbedingt unter streng überwachten Bedingungen zur Anwendung kommen.

Schlüsselwörter

Muskeldystrophie - Duchenne · Spinale

Muskelatrophie $\cdot$ Therapie $\cdot$ Kinder

\section{Molecular therapies in childhood neuromuscular disorders-definite hope versus unknown pitfalls}

\section{Abstract}

Spinal muscular atrophy and muscular dystrophy Duchenne belong to the group of rare neuromuscular diseases manifesting in early childhood. Therapeutic options for some of these rare monogenic diseases have changed significantly in recent years. Molecular therapies such as direct gene transfer or alternative processing of the disease-specific gene play an important role in this transformation.

In particular, the course of $5 q$-associated spinal muscle atrophy has changed significantly due to the availability of such causal therapies, while the results of ongoing studies are still pending for most muscle diseases. In the area of neuromuscular diseases, an achievable therapeutic goal is to slow the progression, but not complete healing. Currently, only limited data are available. In particular, the long-term effectiveness and the possible risks are still unknown. Therefore, these therapies should be used under strictly monitored conditions.

\section{Keywords}

Muscular dystrophy · Duchenne - Spinal muscular atrophy · Children · Therapy gen im Kindesalter. Die weltweite Prävalenz wird mit 1:11.000 Lebendgeburten angegeben [9]. Bei sehr unterschiedlichem klinischen Schweregrad der Unterformen basiert die Erkrankung stets auf einer homozygoten Deletion des Exons $7 \mathrm{im} \mathrm{Gen} \mathrm{für} \mathrm{das} \mathrm{Protein} \mathrm{Survival}$ Motor Neuron 1 (SMN1), das essenziell für das Überleben der Motoneuronen ist. Zudem existiert ein paraloges Gen (SMN2) in unmittelbarer Nähe. Es unterscheidet sich vom SMN1-Gen durch einen kritischen C-zu-T-Basenaustausch im Exon 7, der zu einer dysfunktionalen Translation des betroffenen Exons führt.
Physiologisch werden somit nur 5-10\% der Messenger-RNA (mRNA) aus SMN2 korrekt gebildet und zu funktionsfähigem Protein translatiert. Die Kopienanzahl des paralogen SMN2-Gens gilt als der wichtigste Modifikator für die Erkrankungsschwere der SMA. Patienten mit „nur“ 2 SMN2-Kopien erleiden in den meisten Fällen die schwerste Form der Erkrankung und Versterben zumeist noch im Säuglingsalter am Versagen der Atemhilfsmuskulatur [10]. 


\section{Antisense-Oligonukleotid- Technologie}

Das ASO Nusinersen blockiert eine intronische Gensequenz, die das Spleißen bremst (Splicing Silencer Site). Dies geschieht auf mRNA-Ebene im Exon 7 des SMN2-Gens (• Abb. 1a). Damit wird eine Inklusion von Exon 7 bei der Proteinbiosynthese erreicht, wodurch eine größere Menge an funktionsfähigem SMN-Protein zur Verfügung steht. Aufgrund der positiven Daten der Phase-3Studien (ENDEAR/CHERISH) sowohl bei schwerstbetroffenen (ENDEAR, Manifestation vor 6. Lebensmonat) als auch bei nichtgehfähigen kindlichen Patienten (CHERISH, Manifestation nach dem 6. Lebensmonat, sitzend) erfolgte 2016 bzw. 2017 die amerikanische bzw. europäische Zulassung der Substanz für alle Patienten mit genetisch gesicherter Erkrankung. Symptomatische Patienten zeigten in $47 \%$ bis zu $51 \%$ eine Verbesserung der motorischen Scores und einen positiven Einfluss auf Überleben bzw. die Notwendigkeit einer Beatmung $[11,12]$.

Seit der Zulassung zeigen Daten aus der präsymptomatischen Therapiestudie NURTURE sowie weiteren Publikationen aus Pilotprojekten von Neugeborenenscreenings eine signifikant bessere motorische Entwicklung von vor Erkrankungsbeginn behandelten Säuglingen im Vergleich zu nach Symptombeginn behandelten Kindern [13, 14], sodass für die Experten die Notwendigkeit eines Neugeborenenscreenings für die Erkrankung mittlerweile außer Frage steht.

Die Behandlung mit Nusinersen erfolgt intrathekal. Nach einer Aufdosierungsphase (mit insgesamt 4 Applikationen innerhalb von 2 Monaten) erfolgen Erhaltungsdosen alle 4 Monate.

Relevante Nebenwirkungen sind vor allem durch die Lumbalpunktion (ggf. auch die Strahlenbelastung, falls Durchleuchtung für die Applikation nötig ist) bedingt. Daneben gibt es seit der Zulassung einige Berichte über das weltweite, sporadische Auftreten eines kommunizierenden Hydrocephalus, vor allem bei Säuglingen [15].

Nusinersen gilt als eines der teuersten Arzneimittel weltweit mit Arzneimittel- kosten von ca. $500.000 €$ im ersten Jahr und ca. $300.000 €$ jährlich unter Erhaltungstherapie.

\section{„Kleine Moleküle“}

Die erste oral verabreichbare Substanz ist Risdiplam. Sie führt zu einer Modifikation der Prä-mRNA und damit zu einer Erhöhung der Transkriptionsrate von SMN2. Risdiplam befindet sich nach Erreichen der primären Endpunkte in den Studien SUNFISH (NCT02908685) bzw. FIREFISH (NCT02913482) im beschleunigten Zulassungsprozess bei der US-amerikanischen Food and Drug Administration (FDA), deren Entscheidung im ersten Halbjahr 2020 erwartet wird. Klinische Daten der JEWELFISH-Studie bei vorbehandelten Patienten liegen aktuell noch nicht vor.

Risdiplam wurde in der SUNFISHStudie bei Patienten im Alter von 2 bis 25 Jahren mit SMA-Typ 2 oder 3 untersucht. Ziel der Studie war es, eine Veränderung gegenüber dem Ausgangswert in der Skala „Motor Function Measure 32“ (MFM-32) nach einem Jahr Behandlung im Vergleich zu Placebo zu erreichen. In die FIREFISH-Studie wurden Säuglinge unter 6 Monaten mit SMA-Typ 1 eingeschlossen. Der primäre Endpunkt der Studie war der Anteil der Säuglinge, die nach 12-monatiger Behandlung mindestens $5 \mathrm{~s}$ lang ohne Unterstützung saßen, bewertet anhand der Skala "Gross Motor Scale of the Bayley Scales of Infant and Toddler Development Third Edition" (BSID-III).

Unter den Säuglingen, die die für den bestätigenden Teil 2 der Studie gewählte Dosis erhielten $(n=17)$, konnten 7 $(41,2 \%)$ mindestens $5 \mathrm{~s}$ lang ohne Unterstützung sitzen.

Die häufigsten Nebenwirkungen in allen Studien waren Fieber, Infektionen (vor allem der Atemwege), Durchfall, Verstopfung, Erbrechen. Laut Herstellerangaben wurden bisher in keiner Risdiplam-Studie behandlungsbezogene Sicherheitsergebnisse, die zu einem Studienabbruch führten, identifiziert (Daten präsentiert beim 24. Annual Meeting of the World Muscle Society, 2019).

Eine weitere orale Substanz (Branaplam) befindet sich seit einigen Jahren in einer klinischen Studie (NCT02268552). Nachdem 2016 ein Rekrutierungsstopp aufgrund tierexperimenteller Daten (Schädigung peripherer Nerven) vorgenommen wurde, wurden die Studienaktivitäten 2017 wieder aufgenommen. Bislang liegen keine Ergebnisse vor.

\section{Genersatztherapie}

Zur Behandlung der 5q-SMA wurde zudem eine auf adenoassoziierten Viren (AAV9) basierende Gentherapie (Onasemnogene Abeparvovec-Xioi) entwickelt, die ein vollständiges humanes SMN1-Gen über das Trägervirus transduziert. Das AAV9-Kapsid zeigt bei systemischer Applikation eine gute ZNS-Verfügbarkeit. Die Phase-1-Studie (NCT02122952) mit 15 erkrankten Säuglingen im Alter von bis zu 6 Monaten, bei denen 2 SMN2-Kopien vorhanden waren, konnte ein verlängertes Überleben sowie ein vermehrtes Erreichen von motorischen Meilensteinen im Vergleich $\mathrm{zu}$ einer historischen Kontrolle dokumentieren [16]. Mit diesen Daten wurde die Substanz im Jahr 2019 in den USA zur Behandlung von Kindern mit SMATyp 1 und 2 SMN2-Kopien bis zum Alter von 2 Jahren zugelassen. Aktuell laufen weltweit weitere Studien mit älteren $\mathrm{Pa}$ tienten und milderem Phänotyp sowie präsymptomatischen Patienten [17]. Die geplante Studie zur intrathekalen Gabe wurde abgebrochen, um die Ergebnisse der präklinischen Daten zu bewerten.

Die Kosten für die Behandlung eines Patienten belaufen sich in den USA auf knapp 2 Mio. Dollar. Hinzukommen die Kosten für Vor- und Nachsorge der Patienten unter Gentherapie. Diese erfordert einen erheblichen Ressourcenaufwand mit äußerster spezieller Expertise sowie höchsten Qualitäts- und Sicherheitsstandards.

Seit 19. Mai 2020 ist auch die euopäische Zulassung erteilt, diese weicht im Zulassungtext jedoch von der amerikanischen ab. In Europa können Patienten mit 5qSMA und einer klinischen Diagnose SMATyp1 bzw. bis zu 3 SMN2 Kopien behandelt werden. 


\section{Muskeldystrophie Duchenne}

Beim männlichen Geschlecht ist die $\mathrm{X}$-chromosomal vererbte Muskeldystrophie Duchenne (DMD) die weltweit häufigste neuromuskuläre Erkrankung im Kindesalter und tritt mit einer Frequenz von 1:3500-5000 Geburten auf. In der überwiegenden Mehrzahl sind große Deletionen im Dystrophingen ursächlich, selten auch Duplikationen. Das Fehlen von Dystrophin führt $\mathrm{zu}$ einer Dysfunktion der Muskelzelle, die sich bei den Patienten in einer zunehmenden Muskelschwäche $\mathrm{ab}$ frühem Kindesalter zeigt. Bei ungefähr $15 \%$ aller Patienten führt eine Nonsense-Mutation zu einem vorzeitigen Stop-Codon. Damit bricht die Dystrophinsynthese am Ribosom vorzeitig $a b$ und das verkürzte, nonfunktionale Molekül wird abgebaut.

\section{Read-Through-Therapie}

Für Patienten mit einem vorzeitigen Stop-Codon (• Abb. 1c) kommt eine Therapie mit dem Wirkstoff Ataluren infrage. Für eine Dosis von $40 \mathrm{mg} / \mathrm{kg}$ Körpergewicht (KG) in 3 Einzeldosen (ED) besteht eine Zulassung im europäischen Raum. Zwei randomisierte kontrollierte Studien zeigten einen positiven Trend in der Wegstrecke der Patienten im 6-Minuten-Gehtest, wenngleich dieser primäre Endpunkt nicht statistisch signifikant erreicht wurde. Sekundäre Subgruppenanalysen erbrachten eine statistisch relevante Veränderung vor allem bei behandelten Patienten in der beginnenden motorischen Abbauphase im Vergleich zur Placebogruppe, sodass im Jahr 2014 die Zulassung des Medikamentes für gehfähige Patienten $\mathrm{ab}$ einem Alter von 5 Jahren gewährt wurde, welche mittlerweile für Kinder ab dem 2. Lebensjahr erweitert wurde [18]. Das Forschungsvorhaben STRIDE Registry (Strategic Targeting of Registries and International Datasets of Excellence, NCT00468832) verfolgt als laufende, multizentrische, beobachtende Studie die Sicherheit und Wirksamkeit des Medikaments Ataluren in der Routineversorgung. Erste Ergebnisse legen einen längeren Erhalt der Lungenfunktion (gemessen als forcierte Vitalkapazität
- FVC >50\%) im Vergleich zu einer historischen Kohorte nahe (Daten präsentiert am 24. International Annual Congress of the World Muscle Society, 2019).

Häufige Nebenwirkungen treten vor allem gastrointestinal auf. Daneben werden oft Kopf- und muskuloskelettale Schmerzen berichtet. Da in einer klinischen Studie des Medikamentes an Patienten mit zystischer Fibrose einige Fälle mit verminderter Nierenfunktion beobachtet wurden, darf Ataluren nicht gleichzeitig mit intravenös angewendeten Aminoglykosiden angewendet werden. Vorsicht sollte auch bei gleichzeitiger Anwendung von UGT1A9-Induktoren (z.B. Antibiotikum Rifampicin) geboten sein. Bei gleichzeitiger Gabe von Corticosteroiden ist keine Dosisanpassung notwendig.

\section{Antisense-Oligonukleotid- Technologie}

Theoretisch ist bei fast $80 \%$ aller Dystrophinmutationen ein Exon-Skipping-Ansatz möglich (• Abb. 1b). Bislang wurden unterschiedlich konstruierte ASO in klinischen Studien untersucht [1]. Das am besten untersuchte Exon-51-Skipping ist bei ca. $13 \%$ aller DMD-Patienten einsetzbar. Aufgrund der hohen Nebenwirkungsrate und der mangelnden therapeutischen Effizienz wurde die Entwicklung des Oligonukleotids Drisapersen als erstes Präparat bei DMD eingestellt [19]. Die neutral geladenen Morpholino-Oligonukleotide (PMO) zeigen eventuell eine bessere Verträglichkeit und eine kleine Studie mit dem Wirkstoff Eteplirsen an 17 Patienten zeigte eine mögliche Verlangsamung der Progredienz der DMD im Vergleich zu einer historischen Kontrollgruppe [20]. Dies führte zur Zulassung der Substanz in den USA, eine Zulassung in Europa wurde wegen noch nicht ausreichender Daten bisher nicht erteilt. Daten aus der laufenden Langzeitbeobachtung zeigen einen besseren Erhalt der Lungenfunktion im Vergleich zur historischen Kontrollgruppe, aktuell laufen noch weitere konfirmatorische Studien, betreffend Exon 51, Exon 45 und Exon 53 [7, 8]. Als häufigste Nebenwirkungen werden Kopfschmerzen, proze- durale Schmerzen (bei der Katheteranlage zur wöchentlichen Infusion), Erbrechen sowie Proteinurie berichtet [20]. Kürzlich wurde die Zulassung für Exon53-Skipping (Golodirsen) in den USA erteilt.

Die Erwartung an die nächste Generation der ASOs muss vor allem eine höhere therapeutische Effizienz mit reduzierten Dosisintervallen sowie einem nicht verschlechterten Sicherheitsprofil sein.

\section{Genersatztherapie}

Genersatztherapie mit adenoassoziierten Viren (AAV) stellen eine therapeutische Option bei DMD dar. Die Größe des Dystrophingens übersteigt allerdings die Kapazität der AAV, sodass verkürzte „Minioder Mikrodystrophingene" entwickelt wurden. Die Entwicklung orientierte sich an Patienten mit großen Deletionen, die den milderen Becker-Phänotyp zeigen [21]. Aktuell befinden sich mehrere AAVMini-/Mikrodystrophinkonstrukte in der (prä)klinischen Erprobung [1, 8, 22]. Diese verkürzten (trunkierten) Konstrukte besitzen keine vollständige Dystrophinfunktion, daher kann bestenfalls eine Milderung (Mitigation) des Phänotyps erwartet werden.

Präklinische Daten zeigen eine robuste Expression des verkürzten Dystrophins im Skelettmuskel, mehr als im Myokard. Interimsergebnisse der laufenden Phase-1/2-Studie mit insgesamt $4 \mathrm{~Pa}$ tienten (NCT03375164) zeigen eine effiziente muskuläre Expression, eine Reduktion des Enzyms Creatinkinase sowie verbesserte funktionelle Ergebnisse (Daten präsentiert beim 23. Annual Meeting of the World Muscle Society, 03.10.2018, Mendoza, Argentinien). Häufigste Nebenwirkungen (Leberwerterhöhungen) der AAV-basierten Gentherapien werden auch hier berichtet. Weitere Ergebnisse sind $2021 \mathrm{zu}$ erwarten.

Weitere Mini- (NCT03362502) und Mikrodystrophinkonstrukte (IGNITE DMD, NCT 03368742) befinden sich aktuell in Phase-1- bzw. Phase-1/2-Studien. Für das Minidystrophinkonstrukt (NCT03362502) werden erste Ergebnisse 2020 erwartet. 
Präliminäre Ergebnisse der IGNITEDMD-Studie (NCT 03368742) zeigen, dass die ersten 3 dosierten Patienten bis zu $10 \%$ Expression zeigten. Aktuell befindet sich die Studie aufgrund einer Weisung der FDA in Wartestellung. Ausgelöst wurde diese durch berichtete schwere Nebenwirkungen bei einem 7-jährigen Patienten, der eine Reduktion von Thrombozyten, Erythrozyten, einen akuten Nierenschaden sowie Immunreaktionen (Komplementaktivierung) zeigte.

\section{Weitere Muskelerkrankungen}

\section{Genersatztherapie bei myotubulärer Myopathie und Gliedergürteldystrophien}

Für die kausale Therapie der myotubulären Myopathie (MTM) wurde eine AAV8-basierte systemische Therapie bis zur Phase 1/2 entwickelt. Erste Zwischenergebnisse zeigen eine robuste Expression von Myotubularin im Muskel der Patienten, Erreichen motorischer Meilensteine sowie eine deutliche Verbesserung der Ventilation (Daten präsentiert beim 24. International Annual Congress of the World Muscle Society, 05.10.2019). Die bislang bekannten Nebenwirkungen sind typisch für die AAV-Therapie (Leberwert- und Myokardenzymanstieg, Thrombozytenabfall). Erste finale Ergebnisse werden 2020 erwartet.

Für einige Gliedergürteldystrophien (LGMD 2B, 2C, 2D, 2E, 2I) mit Mutation von Strukturproteinen des Muskels wurden bis dato systemische AAV-basierte Genersatztherapien bis zur Phase $1 / 2$ entwickelt. Erste humane Ergebnisse werden frühestens im Verlauf des Jahres 2020 erwartet.

\section{Fazit}

- Die neuen molekularen Therapien stellen eine große Chance zur Behandlung der seltenen neuromuskulären Erkrankungen dar.

- Als erreichbareres Therapieziel muss insbesondere im Bereich der neuromuskulären Erkrankungen eine Verlangsamung der Progression, aber nicht die vollständige Heilung angesehen werden.
- Die neuen Therapien ersetzen nicht die multidisziplinäre Behandlung, vielmehr machen sie diese noch notwendiger und aufwendiger, um (Neben-)Wirkungen zu dokumentieren und standardisiert in Registern zu untersuchen.

- Aktuell sind nur limitierte Erfahrungen aus klinischen Studien und einzelnen Registern vorhanden. Insbesondere die Fragen der langfristigen Effektivität und des Nebenwirkungsspektrums sind bis dato noch unbekannt. Daher sollten diese Therapien unbedingt unter streng kontrollierten Bedingungen zur Anwendung kommen.

- Die Verbesserung der therapeutischen Effizienz und die Reduktion immunogener Nebenwirkungen sowie deren Verständnis sind die wichtigsten Aufgaben in der Entwicklung zukünftiger molekularer Therapien.

\section{Korrespondenzadresse}

\section{PD Dr. Astrid Blaschek}

Lindwurmstraße 4, 80337 München, Deutschland

astrid.blaschek@med.uni-muenchen.de

Funding. Open Access funding provided by Projekt DEAL.

\section{Einhaltung ethischer Richtlinien}

Interessenkonflikt. A. Blaschek gibt folgende Interessenskonflikte an: Studienärztin für die Studien Embrace-Studie (Nusinersen), Drisapersen-Studien, ASPIRO-Studie (MTM).K. Vill gibtfolgende Interessenskonflikte an: Studienärztin für die Studien EmbraceStudie (Nusinersen), Drisapersen-Studien, ASPIROStudie (MTM). W. Müller-Felber gibt folgende Interessenskonflikte an: Studienarzt für die Studien EmbraceStudie (Nusinersen), Drisapersen-Studien, ASPIROStudie (MTM) sowie Tätigkeiten im Advisory Board für Avexis, Audentes, Roche, PTC, Sarepta, Cytokinetics. U. Schara gibt folgende Interessenskonflikte an: Studienärztin für Duchenne- und SMA-Studien, NHS, MTM1 sowie Tätigkeiten im Advisory Board bzw. Vortragshonorare von Avexis/Novartis, Sarepta, Roche.

Für diesen Beitrag wurden von den Autoren keine Studien an Menschen oder Tieren durchgeführt. Für die aufgeführten Studien gelten die jeweils dort angegebenen ethischen Richtlinien.

Open Access. Dieser Artikel wird unter der Creative Commons Namensnennung 4.0 International Lizenz veröffentlicht, welche die Nutzung, Vervielfältigung,
Bearbeitung, Verbreitung und Wiedergabe in jeglichem Medium und Format erlaubt, sofern Sie den/die ursprünglichen Autor(en) und die Quelle ordnungsgemäß nennen, einen Link zur Creative Commons Lizenz beifügen und angeben, ob Änderungen vorgenommen wurden.

Die in diesem Artikel enthaltenen Bilder und sonstiges Drittmaterial unterliegen ebenfalls der genannten Creative Commons Lizenz, sofern sich aus der Abbildungslegende nichts anderes ergibt. Sofern das betreffende Material nicht unter der genannten Creative Commons Lizenz steht und die betreffende Handlung nicht nach gesetzlichen Vorschriften erlaubt ist, ist für die oben aufgeführten Weiterverwendungen des Materials die Einwilligung des jeweiligen Rechteinhabers einzuholen.

Weitere Details zur Lizenz entnehmen Sie bitte der Lizenzinformation auf http://creativecommons.org/ licenses/by/4.0/deed.de.

\section{Literatur}

1. Shimizu-Motohashi $\mathrm{Y}$, Komaki $\mathrm{H}$, Motohashi $\mathrm{N}$ et al (2019) Restoring dystrophin expression in Duchenne muscular dystrophy: current status of therapeutic approaches. J Pers Med 9(1):1. https:// doi.org/10.3390/jpm9010001

2. Haery L, Deverman BE, MathoKSetal (2019) Adenoassociated virus technologies and methods for targeted neuronal manipulation. Front Neuroanat 13:93

3. Lee D, Liu J, Junn HJ et al (2019) No more helper adenovirus: production of gutless adenovirus (GLAd) free of adenovirus and replicationcompetent adenovirus (RCA) contaminants. Exp Mol Med 51(10):127

4. Petry H, Brooks A, Orme A et al (2008) Effect of viral dose on neutralizing antibody response and transgene expression after AAV1 vector readministration in mice. Gene Ther 15(1):54-60

5. Shorrock HK, Gillingwater TH, Groen EJN (2018) Overview of current drugs and molecules in development for spinal muscular atrophy therapy. Drugs 78(3):293-305

6. Saffari A, Weiler M, Hoffmann GF et al (2019) Gene therapies for neuromuscular diseases. Nervenarzt 90(8):809-816

7. Pulst SM (2019) Antisense therapies for neurological diseases. Nervenarzt 90(8):781-786

8. Mercuri E, Bonnemann CG, Muntoni F (2019) Muscular dystrophies. Lancet 394(10213):2025-2038

9. Sugarman EA, Nagan N, Zhu H et al (2012) Panethnic carrier screening and prenatal diagnosis for spinal muscular atrophy: clinical laboratory analysis of $\rangle 72,400$ specimens. Eur J Hum Genet 20(1):27-32

10. Calucho M, Bernal S, Alias L et al (2018) Correlation between SMA type and SMN2 copy number revisited: an analysis of 625 unrelated Spanish patients and a compilation of 2834 reported cases. Neuromuscul Disord 28(3):208-215

11. Mercuri E, Darras BT, Chiriboga CA et al (2018) Nusinersen versus sham control in later-onset spinal muscular atrophy. NEngl J Med 378(7):625-635

12. Chiriboga CA, Swoboda KJ, Darras BT et al (2016) Results from a phase 1 study of nusinersen (ISISSMN $(R x)$ ) in children with spinal muscular atrophy. Neurology 86(10):890-897

13. Vill K, Kolbel H, Schwartz O et al (2019) One year of newborn screening for SMA — results of a German pilot project. J Neuromuscul Dis 6(4):503-515 
14. De Vivo DC, Bertini E, Swoboda KJ et al (2019) Nusinersen initiated in infants during the presymptomatic stage of spinal muscular atrophy: interim efficacy and safety results from the phase 2 NURTURE study. Neuromuscul Disord 29(11):842-856

15. Bundesinstitut für Arzneimittel und Medizinprodukte (BfArM) (2018) Rote-Hand-Brief zu Spinraza ${ }^{\circledR}$ (Nusinersen): Berichte über das Auftreten eines kommunizierenden Hydrozephalus, der nicht mit einer Meningitis oder Blutung in Verbindung steht. https://www.bfarm.de/SharedDocs/ Risikoinformationen/Pharmakovigilanz/DE/RHB/ 2018/rhb-spinraza.html.Zugegriffen: 14.05.2020

16. Mendell JR, Al-Zaidy S, Shell R et al (2017) Singledose gene-replacement therapy for spinal muscular atrophy. N Engl J Med 377(18):1713-1722

17. ClinicalTrials.gov (2020) Homepage. www. clinicaltrials.gov.Zugegriffen: 14.05.2020

18. Bushby K, Finkel R, Wong B et al (2014) Ataluren treatment of patients with nonsense mutation dystrophinopathy. Muscle Nerve 50(4):477-487

19. Goemans NM, Tulinius $M$, van den Hauwe $M$ et al (2016) Long-term efficacy, safety, and pharmacokinetics of drisapersen in Duchenne muscular dystrophy: results from an open-label extension study. PLoSONE 11(9):e161955

20. Mendell JR, Goemans N, Lowes LP et al (2016) Longitudinal effect of eteplirsen versus historical control on ambulation in Duchenne muscular dystrophy. Ann Neurol 79(2):257-271

21. Wells DJ, Wells KE, AsanteEA etal (1995) Expression of human full-length and minidystrophin in transgenic mdx mice: implications for gene therapy of Duchenne muscular dystrophy. Hum Mol Genet 4(8):1245-1250

22. Duan D (2018) Systemic AAV micro-dystrophin gene therapy for Duchenne muscular dystrophy. MolTher 26(10):2337-2356 